\title{
Divergences in learning and memory among wild zebrafish: Do sex and body size play a role?
}

\author{
Tamal Roy ${ }^{1} \cdot$ Anuradha Bhat ${ }^{1}$ (D)
}

Published online: 19 October 2017

(C) Psychonomic Society, Inc. 2017

\begin{abstract}
Given its diverse ecological distribution, zebrafish has great potential for investigations on the effect of habitat characteristics on cognition. Studies were conducted on four wild-caught zebrafish populations to understand the role of native habitat, sex, and body size in determining learning through a novel task associated with a food reward. The habitat variables, namely, the relative abundances of zebrafish and predatory fish and the substrate and vegetation diversity, were quantified during fish sampling. Fish were subjected to a novel task to find a food reward in a maze over successive training trials followed by a test for memory. Performances of subjects were based on time taken to find the food reward and number of mistakes made during trials, and tests for memory. The experiments revealed significant differences in learning rates and memory across populations. Males made significantly fewer mistakes than females only within two populations. No relationship between performance and body size was observed. The differences in learning and memory among wild zebrafish could be due to differences in predation, complexity, and stability of the native habitats. These findings suggest the possible role of multiple interacting factors in determining learning and memory among populations and point to a need for incorporating effects of several factors in future studies.
\end{abstract}

Electronic supplementary material The online version of this article (https://doi.org/10.3758/s13420-017-0296-8) contains supplementary material, which is available to authorized users.

Anuradha Bhat

anuradhabhat@iiserkol.ac.in

1 Department of Biological Sciences, Indian Institute of Science Education and Research Kolkata, Mohanpur, West Bengal 741 246, India
Keywords Population differences $\cdot$ Learning $\cdot$ Memory . Sex $\cdot$ Zebrafish

Cognitive studies in higher vertebrates have most often focused on investigating differences among closely related species (Shettleworth, 2009). In fish, populations of the same species can be found in greatly contrasting ecological conditions, and this provides ample opportunities to undertake comparative studies on learning within species (Brydges, Heathcote, \& Braithwaite, 2008; White \& Brown, 2014). Previous studies in fish have attempted to understand variations in learning and memory in light of the heterogeneity and stability of habitats (Odling-Smee \& Braithwaite, 2003) and predation pressure (Brown \& Braithwaite, 2005; Brydges et al., 2008). Studies on three-spined sticklebacks (Gasterosteus aculeatus), for example, revealed that pond and river populations differed in the type of spatial information used for learning, with pond fish relying on using visual cues more than river fish (Girvan \& Braithwaite, 1998, 2000; Odling-Smee \& Braithwaite, 2003). A comparison between spatial learning abilities of benthic and limnetic species of three-spine sticklebacks showed that benthic species learn the spatial task twice as quickly as limnetic species (OdlingSmee, Boughman, \& Braithwaite, 2008). It has been noted that learning and memory retention can be shaped by different ecological factors and their interactions. Brydges et al. (2008) showed that low-predation river populations learned a spatial task faster than high-predation river populations, and that river fish had longer memory retention than pond fish. They speculated that high-predation fish might need to divide their attention for other activities, like predator vigilance. This would leave them with less time for exploring potential feeding sites. Further, river fish would have a higher chance of relocation to new areas due to either the flow of the 
river or to exploration, and it would benefit them to have more extensive memory to allow them to relocate to recently explored areas (Brydges et al., 2008). Similarly, populations of the tropical poeciliid Brachyraphis belonging to habitats with low-predation pressures solved a spatial task nearly twice as fast as those from high-predation habitats (Brown \& Braithwaite, 2004). These studies point to the role of multiple ecological factors and their interactions in shaping learning and memory processes in fish. Few studies, however, have attempted to quantify the various ecological factors that could explain the divergences observed in learning.

Cognitive abilities in fish species could be governed by factors other than predation and structural complexity of habitats. For example, competition for access to food resources among conspecifics in the native habitat could determine how quickly individuals would learn the location of food reward in a maze (Brown \& Braithwaite, 2004). Individual factors like body size and sex may also influence learning abilities among fish. A previous study in Xenotoca eiseni had shown sex differences in the use of geometric and nongeometric cues for spatial reorientation (Sovrano, Bisazza, \& Vallortigara, 2003). Sex differences in spatial performance have been shown to correlate with differences in size of the dorsolateral ventral region of the telencephalon, which is involved in the processing of spatial learning and memory (Costa et al., 2011). However, studies in guppies have shown that males and females differed in speed but not in accuracy in visual discrimination learning (Lucon-Xiccato \& Bisazza, 2016). In addition to sex, learning capacities have been found to be related to age and thus size among some species of fish (Makino, Masuda, \& Tanaka, 2006; Masuda \& Ziemann, 2000).

In a previous work, we compared learning and memory abilities among wild and aquarium-bred juveniles (F1) of zebrafish (Danio rerio; Roy \& Bhat, 2016). Here, we aim to investigate learning and memory among individuals from four natural populations of zebrafish. Zebrafish inhabit a diverse range of ecological habitats (Arunachalam, Raja, Vijayakumar, Malaiammal, \& Mayden, 2013; Roy \& Bhat, 2015, 2016; Roy, Shukla \& Bhat, 2017; Suriyampola et al., 2016), and this feature makes the species particularly amenable to experiments testing possible differences in learning and memory across populations. Although domesticated (commercial and laboratory-reared) strains of zebrafish have been used in behavioral studies, investigations on natural populations of the species are rare. Importance of environmental enrichment during early rearing in performance in a maze has been studied in zebrafish juveniles (Roy \& Bhat, 2016) and adults (Spence, Magurran, \& Smith, 2011). However, comparative studies on learning abilities and memory retention on wild zebrafish populations from differing habitat and ecological conditions have not yet been conducted.

We collected four populations across India and simultaneously estimated the relative abundance of zebrafish, total relative abundance of predatory fish, and the complexity (diversity of the substrate and vegetation) of the native habitats. Individual fish were subjected to a novel task to find a food reward over successive training trials. Variations in maze performance and errors committed while finding the reward chamber through the training session were compared among individuals across populations, taking into consideration the sex and body size of individuals. The ability to remember the learned task was tested after a 4-day no-trial gap. The variations observed in learning are explained on the basis of the ecological estimates of the native habitats.

\section{Method}

\section{Populations}

We collected four populations of zebrafish: Seripetkalwa (SK), Kaushalya (KA), Asan (AS), and Wahsympoh (WA) from the states of Andhra Pradesh, Haryana, Uttarakhand, and Meghalaya in India, during the premonsoon season (March-May) of 2013 (Roy, Shukla \& Bhat, 2017). The details for habitat measurements of the four collection sites are illustrated in Table 1.

\section{Measurement of ecological variables}

We measured the physical characteristics of the water bodies (substrate and vegetation diversity) and conducted community sampling to estimate the biotic factors (abundance of zebrafish and predators; see Table 1). In order to maintain a uniform sampling effort, we collected our data on zebrafish and predator (piscivorous fish species) abundances based on 15 cast nets and 10 drag nets employed for each site. We then estimated the abundance of zebrafish and predators relative to the overall abundance of fish species at each site (for the same sampling effort; see Table 1).

Habitat complexity could be defined as the existence of different kinds of elements that constitute a habitat (Tokeshi $\&$ Arakaki, 2012). Fish may use habitats for protection against predation, for spawning, and as shelter. The substratum can be composed of boulders, rocky crevices, submerged plant debris, broken branches, plankton, or be bare. The water column in natural habitats would typically have a combination of live plants as well as floating or partially submerged plant matter. It was thus often difficult to separate these out entirely as part of the substratum or vegetation. We therefore opted to first measure the substratum based on quality and proportion of gravel, sand, rock, and so forth and combined this with the biotic (vegetated) component to get a single estimate for habitat complexity. We used Simpson's index, a commonly used measure of species diversity, to calculate the diversities of substrate and vegetation. It has been used previously in 
Table 1 Environmental and ecological variables of the four water bodies

\begin{tabular}{llllllll}
\hline Site & $\begin{array}{l}\text { Altitude } \\
(\mathrm{m})\end{array}$ & Flow & $\begin{array}{l}\text { Relative abundance } \\
\text { of zebrafish }\end{array}$ & $\begin{array}{l}\text { Total relative abundance } \\
\text { of predatory fish }\end{array}$ & $\begin{array}{l}\text { Channel substrate } \\
\text { diversity }(1-\mathrm{D}) *\end{array}$ & $\begin{array}{l}\text { Diversity of } \\
\text { vegetation } \\
(1-\mathrm{D}) *\end{array}$ & $\begin{array}{l}\text { Habitat } \\
\text { complexity }\end{array}$ \\
\hline Seripetkalwa (SK) & 5.00 & Low & 0.32 & 0.011 & 0.096 & 0.53 & 0.31 \\
Kaushalya (KA) & 406.60 & High & 0.56 & 0.04 & 0.67 & 0.73 \\
Asan (AS) & 514.20 & Stagnant & 0.49 & 0 & 0.63 & 0.18 \\
Wahsympoh (WA) & 1212.0 & Stagnant & 0.62 & 0.007 & 0.27 & 0.1 & 0.1 \\
\hline
\end{tabular}

*Simpson's index of diversity

\#Combination of channel substrate and vegetation diversity

measurement of spatial heterogeneity (Brown, 2003). Simpson's index of diversity is given by the expression $1-$ D (Whittaker, 1965), where D represents Simpson's index. A section of $100 \mathrm{~m}$ of the water body was selected, spanning the location of sampling of zebrafish. A $0.5 \mathrm{~m} \times 0.5 \mathrm{~m}$ quadrat was randomly placed on the water's surface. First, the substrate material forming the bed of the habitat was classified on the basis of established criteria (Cummins, 1962). Substrate material was classified as sand, silt/mud, gravel, cobbles, boulders, and bedrock, and the percentage cover of each of these was recorded (see Supplementary Material). Based on these values, Simpson's index of diversity was calculated and used as the index for substrate diversity (Willis, Winemiller, \& Lopez-Fernandez, 2005; see Table 1). For quantifying the diversity of vegetation, the plant species present in the habitat were categorized as submerged, emergent, or floating. The percentage cover of each category of plant was recorded, and on the basis of these values (see Supplementary Material), the Simpson's index was then calculated as a measurement of diversity of vegetation for each site (see Table 1). Finally, the measures of the two indices were combined to a single diversity index. The value of the Simpson's index, $1-$ $\mathrm{D}$, ranges between 0 and 1 . Therefore, the maximum value the Simpson's index can take is 1 . We added the values of the diversity index obtained separately for substrate and vegetation and divided the sum by 2 , which is the sum of the maximum values of the index $(1+1)$ that could be obtained in each case. This value was taken as a measure of overall habitat complexity for the collection sites (see Table 1).

\section{Maintenance of wild populations in the laboratory}

We collected approximately 60 fish from each site using drag nets and transported them to the laboratory in oxygenated bags. Fish were maintained in bare housing aquaria $(45.7 \times$ $25.4 \times 25.4 \mathrm{~cm}$ ), consisting of a standard corner filter. Approximately 30 mixed-sex individuals were maintained per aquarium, density being the same for all populations. Holding room temperature was maintained at $25^{\circ} \mathrm{C}$, and lighting condition was set at $14 / 10$-hour light/dark cycle to mimic the natural conditions. Each morning the fish were alternately fed freeze-dried bloodworms or Artemia. The experiments were performed on the fish collected from the wild in order to be able to relate differences in learning to the variations in their habitat measures. The fish were maintained for at least 6 months in the laboratory before the commencement of experiments in order to ensure that only adults were used.

\section{Experiment}

\section{Setup}

A square shaped arena $(44.5-1 \times 44.5-\mathrm{w} \times 21.5-\mathrm{h} \mathrm{cm})$ was constructed for testing the performance of adult zebrafish (Roy \& Bhat, 2017) (see Fig. 1). The square tank consisted of an inner square-shaped layer with sides measuring $28.5 \mathrm{~cm}$. The sides of the inner layer were separated from corresponding sides of the outer wall of the tank by $7.5 \mathrm{~cm}$. The edges of the inner square were connected to the diagonal ends of the tank walls by $10-\mathrm{cm}$ connections, thereby forming four separate chambers. Each of the four chambers had a main door leading to the center of the test arena. Each chamber had three sections separated by two windows (openings) as shown. A removable colored frame (red, yellow, green, or blue) identified each main door. The selection of the colors for doors was based on a previous study in Amarillo fish (de Perera \& Macias Garcia, 2003). Zebrafish possess color vision (Cameron, 2002) and are able to distinguish between different colors (Arthur \& Levin, 2001; Colwill, Raymond, Ferreira, \& Escudero, 2005). A food reward associated with an artificial plant was always placed inside the right-hand section of the red-door chamber (see Fig. 1). Pilot experiments using the same testing arena without a reward did not reveal preferences for a particular colored door or door position by subjects (Mann-Whitney $U$ test, $n=10, p=.1$ ). Hence, the choice of the red-door chamber for reward was purely based on random selection. This choice is also supported by previous studies in zebrafish where the use of a red cue card predicted the presence of food (Sison \& Gerlai, 2010; Williams, White, \& Messer, 2002). The position of food reward and plant within 


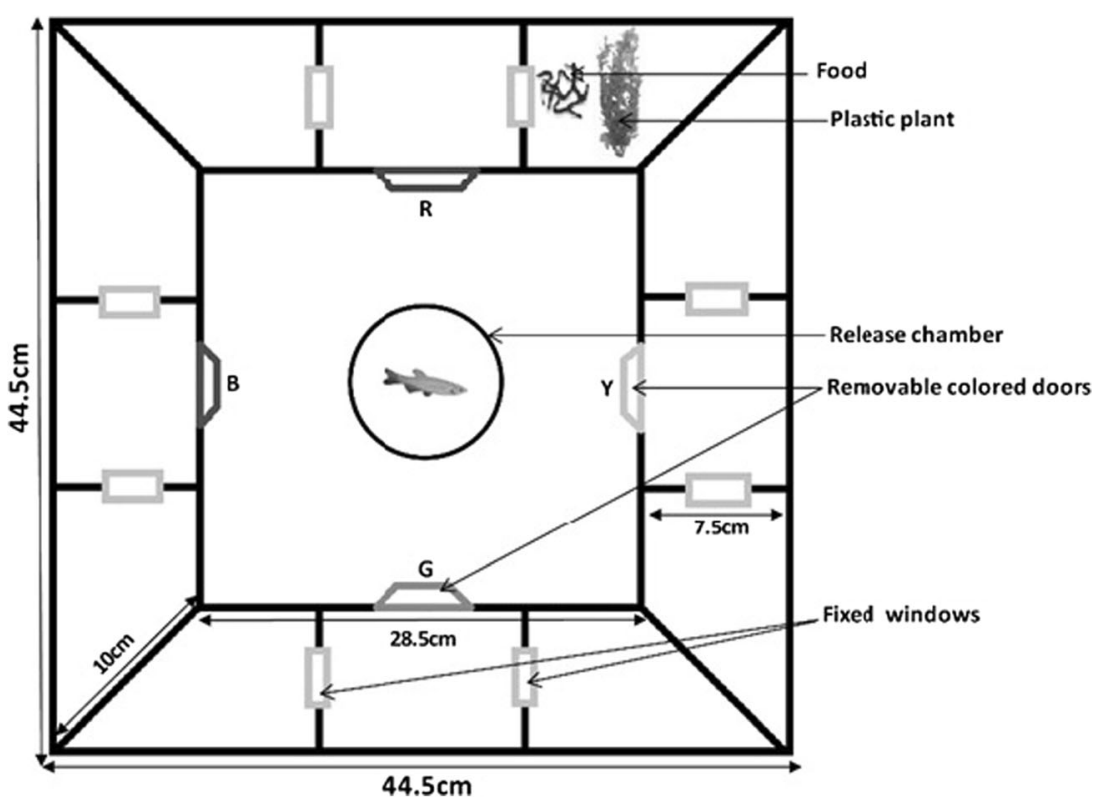

Fig. 1 Schematic diagram for the experimental setup (top view). Square shaped arena has four chambers, each with a colored, removable main

the red-door chamber was fixed and never changed. A test fish would have to explore the maze and locate the food inside the right-side section of the red-door chamber. The task in question therefore was composed of a major color-association component followed by a turn component. The outer wall of the testing tank was covered on all sides with brown cardboard paper to prevent any local external cues from affecting fish behavior. The walls of the inner layer and the diagonal connections were all opaque. The maze was positioned under a white ceiling and uniform lighting conditions.

Each test fish from a population was kept individually in labeled transparent 1 liter cylindrical plastic containers (filled with $520 \mathrm{ml}$ water) throughout the period of training and test (i.e., for 13 days). This method of isolation was necessary to identify fish individually in order to facilitate the tracking of individual behavior over the course of experiments (Roy \& Bhat, 2015, 2016). After the assays, the sex and total length of all individuals (SK, $n=15$; KA, $n=26$; AS, $n=20$ and WA, $n$ $=18$ ) were recorded. All experiments were conducted during the same time of the day, and fish were not fed till the end of all the trials for the day.

\section{Training and test for memory}

The experimental tank was filled with well-aerated water to a depth of $12 \mathrm{~cm}$. A test fish was transferred into an opaque cylindrical release chamber in the middle of the maze and allowed 2 min to acclimatize (see Fig. 1). After this, the release chamber was slowly raised and the fish was allowed to explore the arena. Once the fish entered the red-door chamber and reached the right-hand section, a piece of freeze-dried blood worm was put near the plant, using forceps. The door. Each chamber is divided into three sections by two fixed windows. $\mathrm{R}=$ red; $\mathrm{Y}=$ yellow; $\mathrm{G}=$ green; $\mathrm{B}=$ blue

behavior of the fish was video recorded for 20 min using a high-definition camcorder Canon LEGRIA HF R306 placed at an optimum distance overlooking the experiment setup. We set the maximum duration of a trial at $20 \mathrm{~min}$. After the experiment, the fish was carefully netted out and put back into its respective container for successive trials. The excess uneaten food was siphoned off, and water in the tank was changed to avoid olfactory cues from affecting learning. An individual fish was subjected to a trial only once each day. Each fish was trained through the maze to locate the food reward for 8 consecutive days. The position of the red door, with the plant and the reward, was changed every day but kept constant through all trials for other individuals tested during a day. No 2 consecutive days had the red door in the same position. Following training, an intermission of 4 days was given during which the fish were not subjected to trials. During this period, the fish were retained in their respective containers and were fed normally as in the daily routine. Postintermission, the fish were retested on the 13th day following the same protocol for their ability to remember the learned task.

The video recordings were manually analyzed for (1) time taken by an individual fish to find the food reward and commence feeding on each day from its time of release (denoted as "performance time"), and (2) the number of wrong entries (denoted as "mistakes") made till the discovery of food reward on each day from second trial to the final test. An upper limit of $20 \mathrm{~min}$ was assigned for performance time if an individual failed to find the food reward within the duration. To avoid observer bias, the videos were analyzed in blind (i.e., the population being observed at any given time was unknown to the observer). 
Our studies complied with the existing rules and guidelines outlined by the Committee for the Purpose of Control and Supervision of Experiments on Animals (CPCSEA), Government of India. The studies adhered to the Institutional Animal Ethics Committee's (IAEC) rules and guidelines of IISER Kolkata (Reg. No. 1385/ac/10/ CPCSEA). Zebrafish (Danio rerio) is designated as a species of Least Concern by IUCN's Red List of threatened species. The community sampling and zebrafish collections were not made inside any reserved forest or protected area. The fish caught during community sampling were identified, recorded, and put back into the water bodies. No animals were euthanized or sacrificed during any part of the study, and behavioral observations were conducted without any chemical treatment on individuals. At the end of the experiments, all zebrafish were transferred to stock tanks in the laboratory, where they continue to be maintained.

\section{Statistical analysis}

All data analyses were conducted using StatistiXL Version 1.8 and R software.

Prior to analysis, behavioral data on performance and number of mistakes was square-root transformed to normalize error distribution. Separate linear mixed models (LMMs) were used for performance time and number of mistakes made by individuals (response variables) in $\mathrm{R}$ Version 3.3.1 (R Core Team, 2013) and lme4 (Bates et al., 2012). Sex, body size, population, trial, and interaction effects were taken as fixed effects and individual IDs as random effect, to account for repeated measures of the same individuals across trials. The Satterthwaite method was used to approximate degrees of freedom, and significance level was set at $\alpha<0.05$. Factors that were found to be statistically significant were further analyzed using nonparametric Kruskal-Wallis and MannWhitney tests. In order to test for memory of individuals within a population, the performance time and number of mistakes committed during eighth trial and test was compared using Wilcoxon paired-samples test.

\section{Results}

\section{Comparison of learning and memory across populations}

We found significant effects of trial, $F(7,525)=39.60, p<$ .001 , and Trial $\times$ Population, $\mathrm{F}(21,525)=1.90, p=.009$, from the linear mixed model (LMM) for performance time (see Fig. 2 ). Since we found a significant Trial $\times$ Population interaction effect, we then compared the first, fifth, and last trial (eighth) performances of individuals across populations. We selected the first and the eighth trials as these were the initial and final tests for performance for post hoc comparisons. Additionally we also compared the fifth trial performance as it was roughly halfway through the training. The first trial performance across populations was not significantly different (KruskalWallis test, $\chi^{2}=0.93, p=.82$ ). The fifth trial performances of individuals were significantly different across populations (Kruskal-Wallis test, $\chi^{2}=14, p=.003$ ). Post hoc tests (Mann-Whitney $U$ test, with a Bonferroni correction for multiple comparisons) revealed significant differences between performances of WA and SK $(U=230, p<.001)$, WA and $\mathrm{KA}(U=347, p=.006)$ and WA and $\operatorname{AS}(U=283, p=.002$; see Table 2). Finally, individuals across populations also differed in the eighth, or the last, trial performances (KruskalWallis test, $\left.\chi^{2}=12.96, p=.005\right)$. Post hoc tests revealed significant differences between performances of WA and SK $(U=225, \mathrm{p}=.001)$, and WA and KA $(U=362, p=.002$; see Table 2). Therefore, the performance of population WA was most different from the other three populations during the 5 th and the 8th days of training. The LMM for number of mistakes showed significant effects of trial, $\mathrm{F}(6,450)=5.16, p<$ .001 ; sex, $F(1,70)=0.01, p=.01$; and Population $\times \operatorname{Sex}, F(3$, $70)=3.5, p=.02$; see Fig. 3). Males committed significantly fewer mistakes than females within populations KA $(t=$ $-2.03, p=.04)$ and SK $(t=-2.15, p=.03$; see Fig. 4). In tests for memory based on comparisons of performance and number of mistakes on eighth and the test trial (see Fig. 5), only populations KA and AS indicated strong memory retention abilities (see Table 3).

\section{Discussion}

Our results showed that performance of individuals improved (see Fig. 2) and the number of mistakes made by individuals declined (see Fig. 3) in the maze across trials, indicating learning. The mean performance time declined rapidly over training sessions (trials) for populations KA, AS, and SK, whereas it declined relatively slowly for WA. Individuals across populations differed in their performance rates, as indicated by the significant interaction between population and trial. Though the populations started with a similar initial performance in the maze, their performances varied as trials progressed. The mean performance time of population WA was higher than other populations throughout the training (see Fig. 2) and significantly differed from the performance of other populations during the fifth and eighth trials (see Table 2). Therefore, the performance of WA varied the most among the four populations.

Our findings emphasize the plausible role of environmental factors such as complexity of the native habitat, predation, and flow conditions in explaining differences in performance in a maze. Learning and memory processes are fine-tuned within a population to suit specific environmental requirements that the animals encounter (Brydges et al., 2008). Habitats that are 


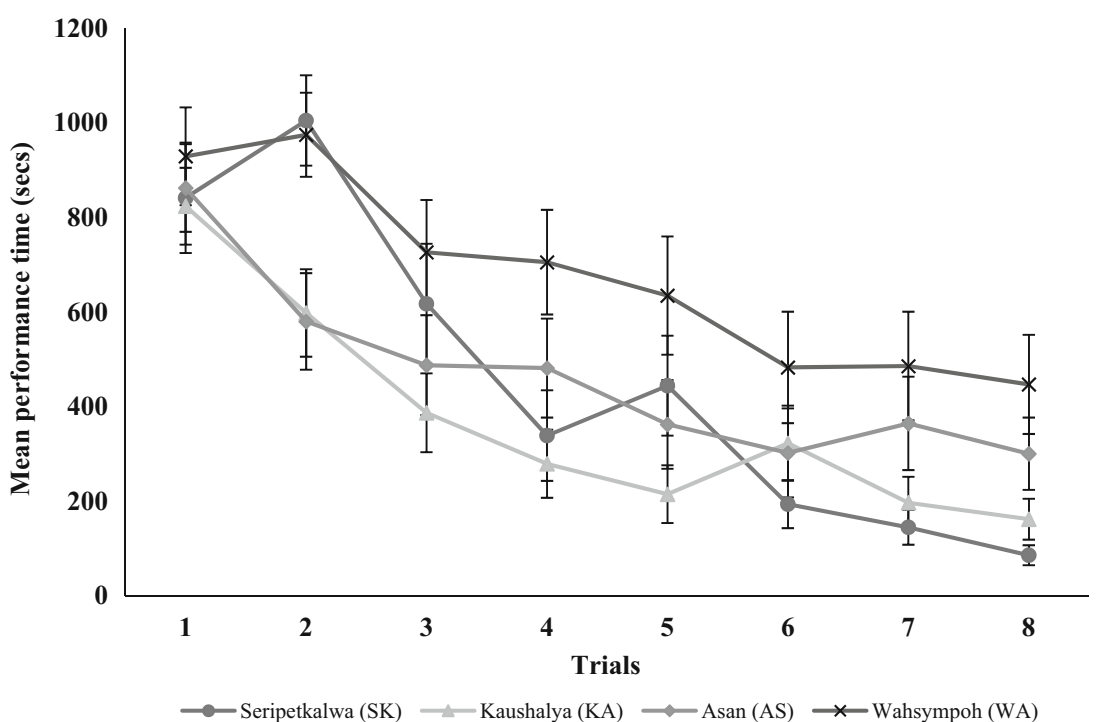

Fig. 2 Mean performance time of individuals across populations Seripetkalwa (SK), Kaushalya (KA), Asan (AS), and Wahsympoh (WA) over eight trials. Data points represent mean $\pm S E$

spatially complex would provide for an enriched rearing environment for development of increased neural plasticity and enhanced cognitive behavioral processes (Salvanes et al., 2013). In our study, the natural habitat of WA population was structurally least complex (see Table 1), and therefore lack of enrichment during early rearing could have resulted in relatively weaker learning abilities. Predation pressure within native habitats also exerts a strong selective force in shaping learning and memory processes (Brown \& Braithwaite, 2005). However, unlike previous studies that showed low-predation populations to have better learning abilities than high-predation populations (Brown \& Braithwaite, 2005; Brydges et al., 2008), we found a lowpredation population (WA) to have shown the "worst" performance in the maze. Therefore, comparative studies with more replication of populations from each kind of habitat are warranted to disentangle the role of each of these factors in shaping learning abilities. It is important to note that the stability of these habitats (Brown \& Braithwaite, 2005; Odling-Smee et al., 2008) and availability or nonavailability of other cues (Rodriguez, Duran, Vargas, Torres, \& Salas, 1994; Roitblat,
Tham, \& Golub, 1982) could also affect cognitive abilities. Rivers and streams experience higher levels of variation in flow conditions because of seasonal flooding or drying (Lytle \& Poff, 2004) and are therefore more unstable compared to pond habitats (Brydges et al., 2008). However, in order to be able to relate habitat instability to differences in learning abilities among zebrafish populations, the reported habitats would need seasonal sampling.

The differences observed in learning could be attributed to confounding effects of other factors as well. Individuals across populations could differ in swimming speed, which could affect the time they took to find the food. Differences in activity and exploratory tendencies between individuals of populations could amount to differences in abilities to solve the spatial task. Bolder individuals could be better at associating a stimulus with a food reward than the shyer ones (Dugatkin \& Alfieri, 2003). Intrinsic metabolic demand has a strong influence on feeding behavior and boldness (Killen, Fu, Wu, Wang, \& Fu, 2016; Metcalfe, Van Leeuwen, \& Killen, 2016) and is likely to play a role in the observed effects. Fish belonging to high-flow habitats have high metabolic rate

Table 2 Post hoc test results for comparison of fifth and eighth trial performances across populations

\begin{tabular}{lll}
\hline Population pairs & 5th trial performance $U(p$ value $)$ & 8th trial performance $U(p$ value $)$ \\
\hline SK and KA & $217.0(.56)$ & $209.0(.72)$ \\
SK and AS & $173.0(.46)$ & $201.0(.09)$ \\
SK and WA & $\mathbf{2 3 0 . 5 ( < . 0 0 1 )}$ & $\mathbf{2 2 5 . 0}(.001)$ \\
KA and AS & $272.5(.78)$ & $317.5(.213)$ \\
KA and WA & $\mathbf{3 4 7 . 0 ( . 0 0 6 )}$ & $\mathbf{3 6 2 . 0}(\mathbf{0 0 2})$ \\
AS and WA & $\mathbf{2 8 3 . 0}(\mathbf{. 0 0 2})$ & $227.0(.17)$
\end{tabular}

Note. Significant results $(p<.05)$ are highlighted in bold. SK = Seripetkalwa; KA = Kaushalya; AS = Asan; WA = Wahsympoh 


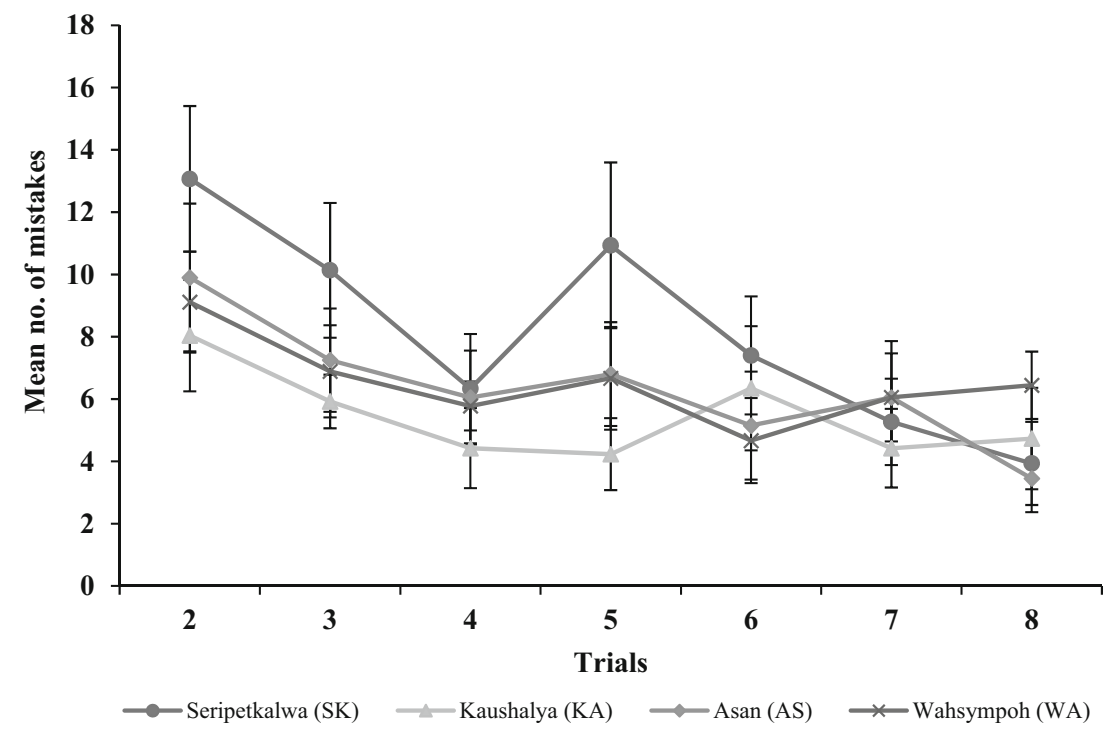

Fig. 3 Mean number of mistakes made by individuals across populations Seripetkalwa (SK), Kaushalya (KA), Asan (AS), and Wahsympoh (WA) over eight trials. Data points represent mean $\pm S E$

and higher energy needs that could result in increased foraging requirement (Killen, Glazier, et al., 2016; Sinclair, de Souza, Ward, \& Seebacher, 2014; Roy, Shukla \& Bhat, 2017). Since
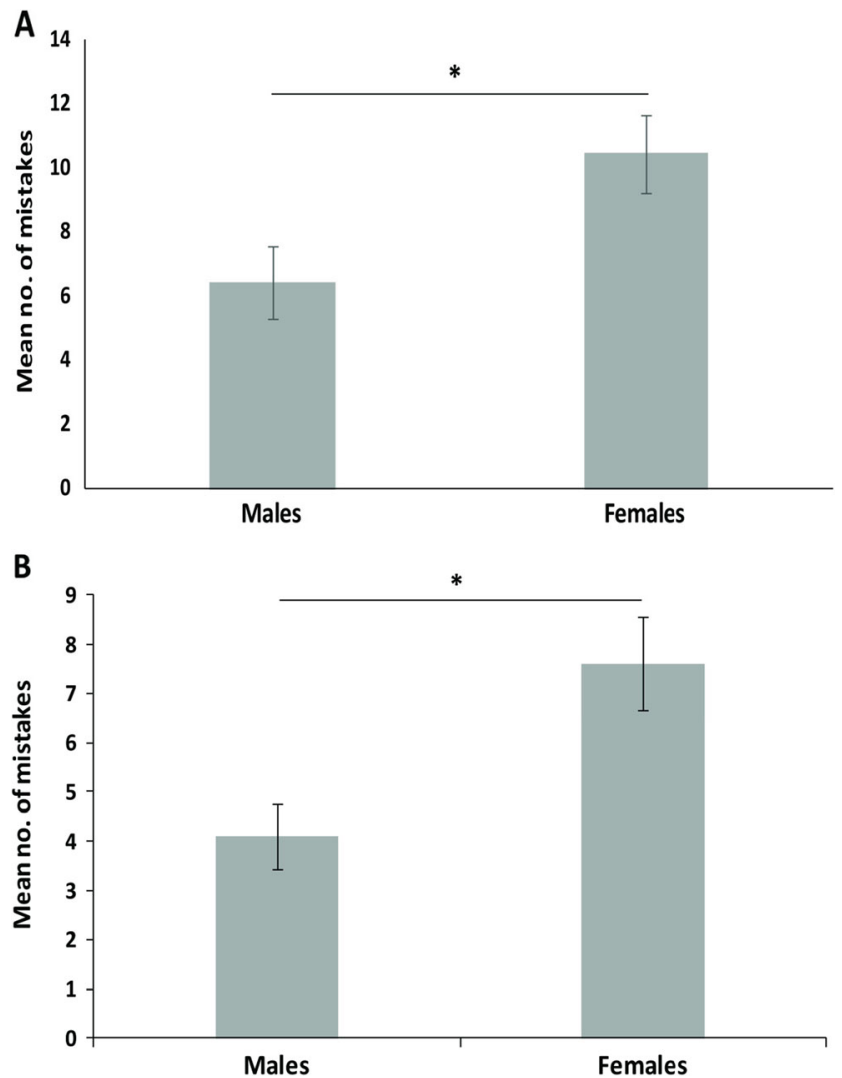

Fig. 4 Comparison of mean number of mistakes made by males and females of (a) Seripetkalwa (SK) and (b) Kaushalya (KA) populations during training. Males made significantly fewer mistakes than females. Statistically significant difference $(\mathrm{p}<0.05)$ is represented by ' $*$ '. Bars represent standard errors the task involved finding a food reward, individuals with higher metabolic rate could be expected to have greater urge to locate the same during subsequent trials. Again, since wildcollected fish could have been of different ages, this could have potentially affected their learning abilities as well. Further, the interpopulation differences could have been mediated by different degrees of cerebral lateralization among populations through turn biases, and therefore some populations could have performed better than the others. Population variations in lateralized eye use has been previously documented in the poeciliid Brachyraphis where high-predation fish viewed predators with the right eye and novel objects with the left, whereas no preference for eye use was observed in low-predation fish (Brown, Gardner, \& Braithwaite, 2004). However, the extent to which differences in brain lateralization among wild zebrafish may translate to turn biases within populations remain to be tested.

Although our results showed no significant effect of sex and body size on performance time, there were sex differences in the number of mistakes made. Males made significantly fewer mistakes than females in two populations, KA and SK. This is in agreement with a study in rats, where males made fewer errors than females while locating a food reward in a radial maze (Seymoure, Dou, \& Juraska, 1996). A recent study in guppies showed that males outperformed females in a relatively complex route learning task (Lucon-Xiccato \& Bisazza, 2017). In our study, differences in number of mistakes committed could be linked to difference in precision and speed with which the color-association task was learned. Further, differences between sexes in laterality or preference for sides (Moscicki \& Hurd, 2015; Reddon \& Hurd, 2009) and color preferences in a foraging context (Rodd et al., 2002) could have resulted in differences in number 

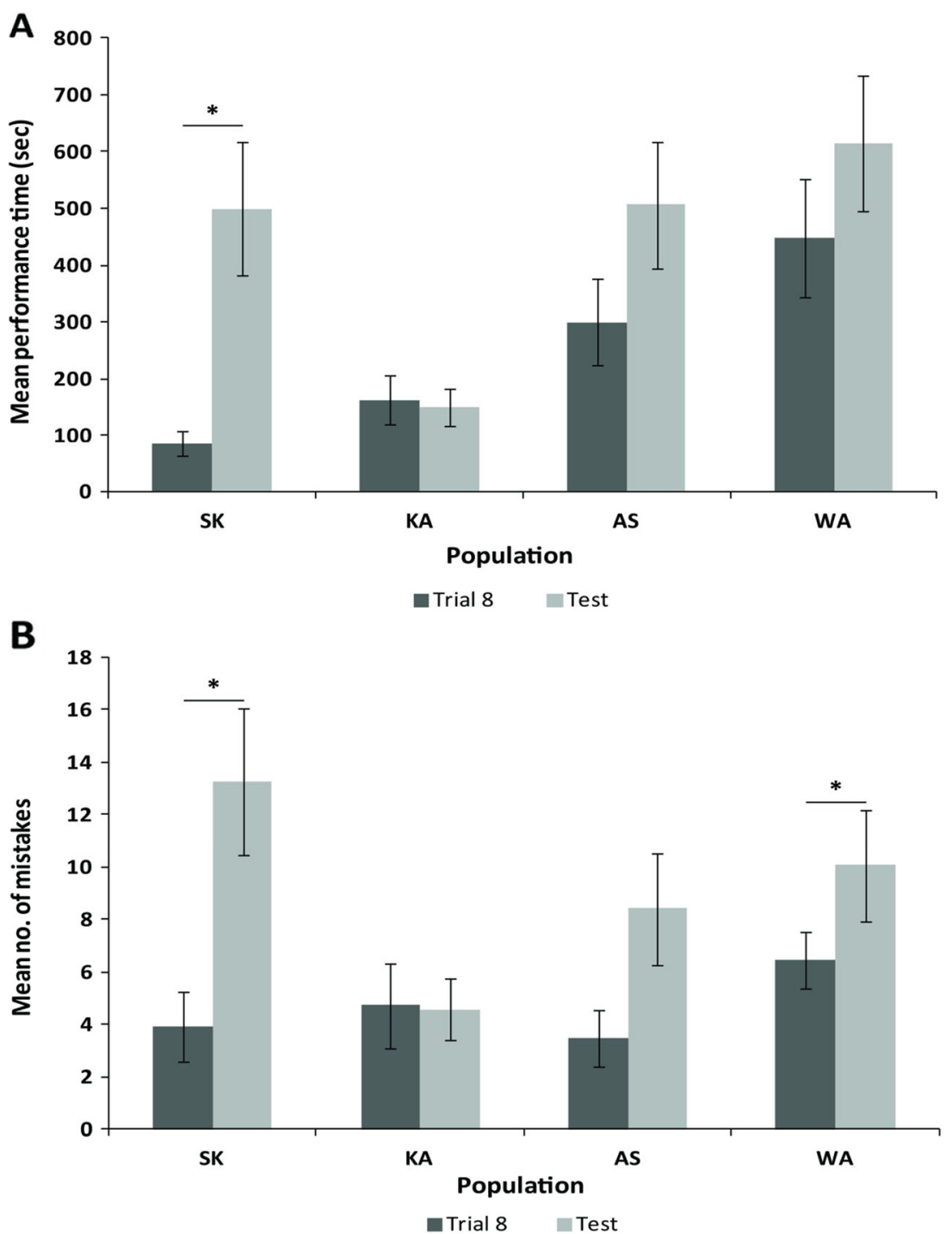

Fig. 5 Tests for memory. Comparison of (a) mean performance time and (b) mean number of mistakes made during the eighth trial and test trial.

of wrong entries made. Such variations could be population specific, and therefore we observed males to have made fewer mistakes than females only within two populations. Further studies testing divergences in laterality and color

Table 3 Results of Wilcoxon paired-sample test for memory

\begin{tabular}{lll}
\hline Population & Performance time T $(p$ value $)$ & Mistakes T $(p$ value $)$ \\
\hline SK $(n=15)$ & $\mathbf{3}(<.001)$ & $\mathbf{4 . 5}(<.001)$ \\
KA $(n=26)$ & $159.50(.69)$ & $148(.58)$ \\
AS $(n=20)$ & $52(.051)$ & $54.5(.08)$ \\
WA $(n=18)$ & $43(.1)$ & $\mathbf{4 0}(\mathbf{. 0 4})$
\end{tabular}

Note. Comparisons were made between performance time and number of mistakes made during eighth and test trial within populations. Significant results $(p<.05)$ are highlighted in bold. SK = Seripetkalwa; $\mathrm{KA}=$ Kaushalya; AS = Asan; WA = Wahsympoh
Statistically significant difference $(\mathrm{p}<0.05)$ is represented by ' $*$ '. Bars represent standard errors

preference between sexes and among wild populations are required to disentangle the interplay of these factors in influencing performance in maze.

In tests for memory, individuals of populations KA and AS showed stronger abilities to remember the learnt task (see Figs. 5a-b). On the other hand, memory of the task was significantly reduced for individuals of the SK population, whereas WA, while having shown weakest learning abilities in the maze, remembered the task. Memory retention is an energetically expensive process and develops in organisms that inhabit environments that demand its requirement (Dukas, 1999; Odling-Smee et al., 2008). Spatially complex habitats would promote development of increased neural plasticity and enhanced cognitive abilities (Salvanes et al., 2013). The ability to learn and remember the association of various cues for locating foraging patches or shelters in composite topographies would be favored by natural selection. Our 
results support the ecological cognition hypothesis, which suggests that environmental factors can play a key role in developing the learning and memory skills (Healy \& Braithwaite, 2000) crucial for survival, and the ability to learn based on ecological cues would be adaptive in nature. Therefore, KA and AS individuals, which occurred in habitats with comparatively greater substrate and vegetation diversity, exhibited stronger memory abilities compared to individuals from SK and WA, which occurred in spatially simple habitats. The temporal stability of habitats with respect to food availability could also determine duration of memory retention among fish (Mackney \& Hughes, 1995). The role of habitat stability in influencing memory was beyond the scope of this study, as habitat information was obtained from a single sampling survey.

In conclusion, our findings suggest that differences in learning and memory among wild zebrafish could be caused by a combination of habitat-related and morphological and physiological factors. The strategies adopted by individuals are likely to be adaptively optimized for survival in the immediate surroundings. Whether these responses are phenotypically plastic or genetically determined and therefore fixed within populations needs to be investigated. Further experiments on heritability of these traits across populations are warranted to tease apart the roles played by either of these factors.

Acknowledgements The Indian Institute of Science Education and Research Kolkata (IISER Kolkata), India, provided financial support during this study. T. Roy was supported by a Senior Research Fellowship from the Council of Scientific and Industrial Research (CSIR), Government of India. The authors express sincere gratitude to Rohitashva Shukla for assistance during collection of wild populations and ecological data from the study locations. The authors also thank the local fishermen for help during collections of zebrafish.

\section{References}

Arthur, D., \& Levin, E. D. (2001). Spatial and non-spatial visual discrimination learning in zebrafish (Danio rerio). Animal Cognition, 4(2), $125-131$.

Arunachalam, M., Raja, M., Vijayakumar, C., Malaiammal, P., \& Mayden, R. L. (2013). Natural history of zebrafish (Danio rerio) in India. Zebrafish, 10, 1-14.

Bates, D., Maechler, M., \& Bolker, B. (2012). lme4: linear mixed-effects models using S4 classes. R package version 0.999375-42. 2011.

Brown, B. L. (2003). Spatial heterogeneity reduces temporal variability in stream insect communities. Ecology Letters, 6, 316-325.

Brown, C., \& Braithwaite, V. A. (2004). Effects of predation pressure on the cognitive ability of the poeciliid Brachyraphis episcopi. Behavioral Ecology, 16, 482-487.

Brown, C., Gardner, C., \& Braithwaite, V. A. (2004). Population variation in lateralized eye use in the poeciliid Brachyraphis episcopi. Proceedings of the Royal Society B: Biological Sciences, 271, S455-S457.

Brydges, N. M., Heathcote, R. J. P., \& Braithwaite, V. A. (2008). Habitat stability and predation pressure influence learning and memory in populations of three-spined sticklebacks. Animal Behaviour, 75, 935-942.

Cameron, D. A. (2002). Mapping absorbance spectra, cone fractions, and neuronal mechanisms to photopic spectral sensitivity in the zebrafish. Visual Neuroscience, 19, 365-372.

Colwill, R. M., Raymond, M. P., Ferreira, L., \& Escudero, H. (2005). Visual discrimination learning in zebrafish (Danio rerio). Behavioural Processes, 70, 19-31.

Costa, S. S., Andrade, R., Carneiro, L. A., Gonçalves, E. J., Kotrschal, K., \& Oliveira, R. F. (2011). Sex differences in the dorsolateral telencephalon correlate with home range size in blenniid fish. Brain, Behavior and Evolution, 77, 55-64.

Cummins, K. W. (1962). An evaluation of some techniques for the collection and analysis of benthic samples with special emphasis on lotic waters. The American Midland Naturalist, 67, 477-504.

de Perera, T. B., \& Macias Garcia, C. (2003). Amarillo fish (Girardinichthys multiradiatus) use visual landmarks to orient in space. Ethology, 109, 341-350.

Dugatkin, L. A., \& Alfieri, M. S. (2003). Boldness, behavioral inhibition and learning. Ethology Ecology \& Evolution, 15, 43-49.

Dukas, R. (1999). Costs of memory: Ideas and predictions. Journal of Theoritical Biology, 197, 41-50.

Girvan, J., \& Braithwaite, V. A. (1998). Population differences in spatial learning in three-spined sticklebacks. Proceedings of the Royal Society B: Biological Sciences, 265, 913-918.

Girvan, J., \& Braithwaite, V. A. (2000). Orientation behaviour in sticklebacks: Modified by experience or population specific? Behaviour, 137, 833-843.

Healy, S., \& Braithwaite, V. (2000). Cognitive ecology: a field of substance? Trends in Ecology \& Evolution, 15(1), 22-26.

Killen, S. S., Fu, C., Wu, Q., Wang, Y.-X., \& Fu, S.-J. (2016). The relationship between metabolic rate and sociability is altered by food deprivation. Functional Ecology, 30, 1358-1365.

Killen, S. S., Glazier, D. S., Rezende, E. L., Clark, T. D., Atkinson, D., Willener, A. S. T., \& Halsey, L. G. (2016). Ecological influences and morphological correlates of resting and maximal metabolic rates across teleost fish species. The American Naturalist, 187, 592-606.

Lucon-Xiccato, T., \& Bisazza, A. (2016). Male and female guppies differ in speed but not in accuracy in visual discrimination learning. Animal Cognition, 19, 733-744.

Lucon-Xiccato, T., \& Bisazza, A. (2017). Sex differences in spatial abilities and cognitive flexibility in the guppy. Animal Behaviour, 123, 53-60.

Lytle, D. A., \& Poff, N. L. (2004). Adaptation to natural flow regimes. Trends in Ecology \& Evolution, 19, 94-100.

Mackney, P., \& Hughes, R. (1995). Foraging behaviour and memory window in sticklebacks. Behaviour, 132, 1241-1253.

Makino, H., Masuda, R., \& Tanaka, M. (2006). Ontogenetic changes of learning capability under reward conditioning in striped knifejaw Oplegnathus fasciatus juveniles. Fisheries Science, 72, 1177-1182.

Masuda, R., \& Ziemann, D. A. (2000). Ontogenetic changes of learning capability and stress recovery in Pacific threadfin juveniles. Journal of Fish Biology, 56, 1239-1247.

Metcalfe, N. B., Van Leeuwen, T. E., \& Killen, S. S. (2016). Does individual variation in metabolic phenotype predict fish behaviour and performance? Journal of Fish Biology, 88, 298-321.

Moscicki, M. K., \& Hurd, P. L. (2015). Sex, boldness and stress experience affect convict cichlid, Amatitlania nigrofasciata, open field behaviour. Animal Behaviour, 107, 105-114.

Odling-Smee, L., \& Braithwaite, V. A. (2003). The influence of habitat stability on landmark use during spatial learning in the three-spined stickleback. Animal Behaviour, 65, 701-707.

Odling-Smee, L. C., Boughman, J. W., \& Braithwaite, V. A. (2008). Sympatric species of threespine stickleback differ in their performance in a spatial learning task. Behavioral Ecology and Sociobiology, 62, 1935-1945. 
Reddon, A. R., \& Hurd, P. L. (2009). Sex differences in the cerebral lateralization of a cichlid fish when detouring to view emotionally conditioned stimuli. Behavioural Processes, 82(1), 25-29.

Rodd, F. H., Hughes, K. A., Grether, G. F., \& Baril, C. T. (2002). A possible non-sexual origin of mate preference: are male guppies mimicking fruit? Proceedings of the Royal Society B: Biological Sciences, 269(1490), 475-481.

Rodriguez, F., Duran, E., Vargas, J. P., Torres, B., \& Salas, C. (1994). Performance of goldfish trained in allocentric and egocentric maze procedures suggests the presence of a cognitive mapping system in fishes. Animal Learning \& Behavior, 22, 409-420.

Roitblat, H. L., Tham, W., \& Golub, L. (1982). Performance of Betta splendens in a radial arm maze. Animal Learning \& Behavior, 10, $108-114$.

Roy, T., \& Bhat, A. (2015). Can outcomes of dyadic interactions be consistent across contexts among wild zebrafish? Royal Society Open Science, 2, 150282.

Roy, T., \& Bhat, A. (2016). Learning and memory in juvenile zebrafish: What makes the difference-population or rearing environment? Ethology, 122, 308-318.

Roy, T., \& Bhat, A. (2017) Social learning in a maze? Contrasting individual performance among wild zebrafish when associated with trained and naive conspecifics. Behavioural Processes, 144, 51-57.

Roy, T., Shukla, R., \& Bhat A. (2017) Risk-taking during feeding: Between- and within population variation and repeatability across contexts among wild zebrafish. Zebrafish. https/doi.org/10.1089/ zeb.2017.1442

Salvanes, A. G. V., Moberg, O., Ebbesson, L. O. E., Nilsen, T. O., Jensen, K. H., \& Braithwaite, V. A. (2013). Environmental enrichment promotes neural plasticity and cognitive ability in fish. Proceedings of the Royal Society of London B: Biological Sciences, 280, 20131331.

Seymoure, P., Dou, H. U. I., \& Juraska, J. M. (1996). Sex differences in radial maze performance: Influence of rearing environment and room cues. Psychobiology, 24, 33-37.
Shettleworth, S. J. (2009). Animal Cognition: Deconstructing Avian Insight. Current Biology, 19(22), R1039-R1040

Sinclair, E. L. E., de Souza, C. R. N., Ward, A. J. W., \& Seebacher, F. (2014). Exercise changes behaviour. Functional Ecology, 28, 652659.

Sison, M., \& Gerlai, R. (2010). Associative learning in zebrafish (Danio rerio) in the plus maze. Behavioural Brain Research, 207, 99-104.

Sovrano, V. A., Bisazza, A., \& Vallortigara, G. (2003). Modularity as a fish (Xenotoca eiseni) views it: Conjoining geometric and nongeometric information for spatial reorientation. Journal of Experimental Psychology: Animal Behavior Processes, 29, 199210.

Spence, R., Magurran, A. E., \& Smith, C. (2011). Spatial cognition in zebrafish: The role of strain and rearing environment. Animal Cognition, 14, 607-612.

Suriyampola, P. S., Shelton, D. S., Shukla, R., Roy, T., Bhat, A., \& Martins, E. P. (2016). Zebrafish social behavior in the wild. Zebrafish, 13(1), 1-8.

Team, R. C. (2013). R foundation for statistical computing. Vienna, Austria, 3(0). http://www.R-project.org/

Tokeshi, M., \& Arakaki, S. (2012). Habitat complexity in aquatic systems: Fractals and beyond. Hydrobiologia, 685, 27-47.

White, G. E., \& Brown, C. (2014). A comparison of spatial learning and memory capabilities in intertidal gobies. Behavioral Ecology and Sociobiology, 68, 1393-1401.

Whittaker, R. H. (1965). Dominance and diversity in land plant communities. Science, 147, 250-260.

Williams, F. E., White, D., \& Messer, W. S. (2002). A simple spatial alternation task for assessing memory function in zebrafish. Behavioural Processes, 58, 125-132.

Willis, S. C., Winemiller, K. O., \& Lopez-Fernandez, H. (2005). Habitat structural complexity and morphological diversity of fish assemblages in a Neotropical floodplain river. Oecologia, 142, 284-295. 